American Journal of

Health, Medicine and Nursing Practice (AJHMN)

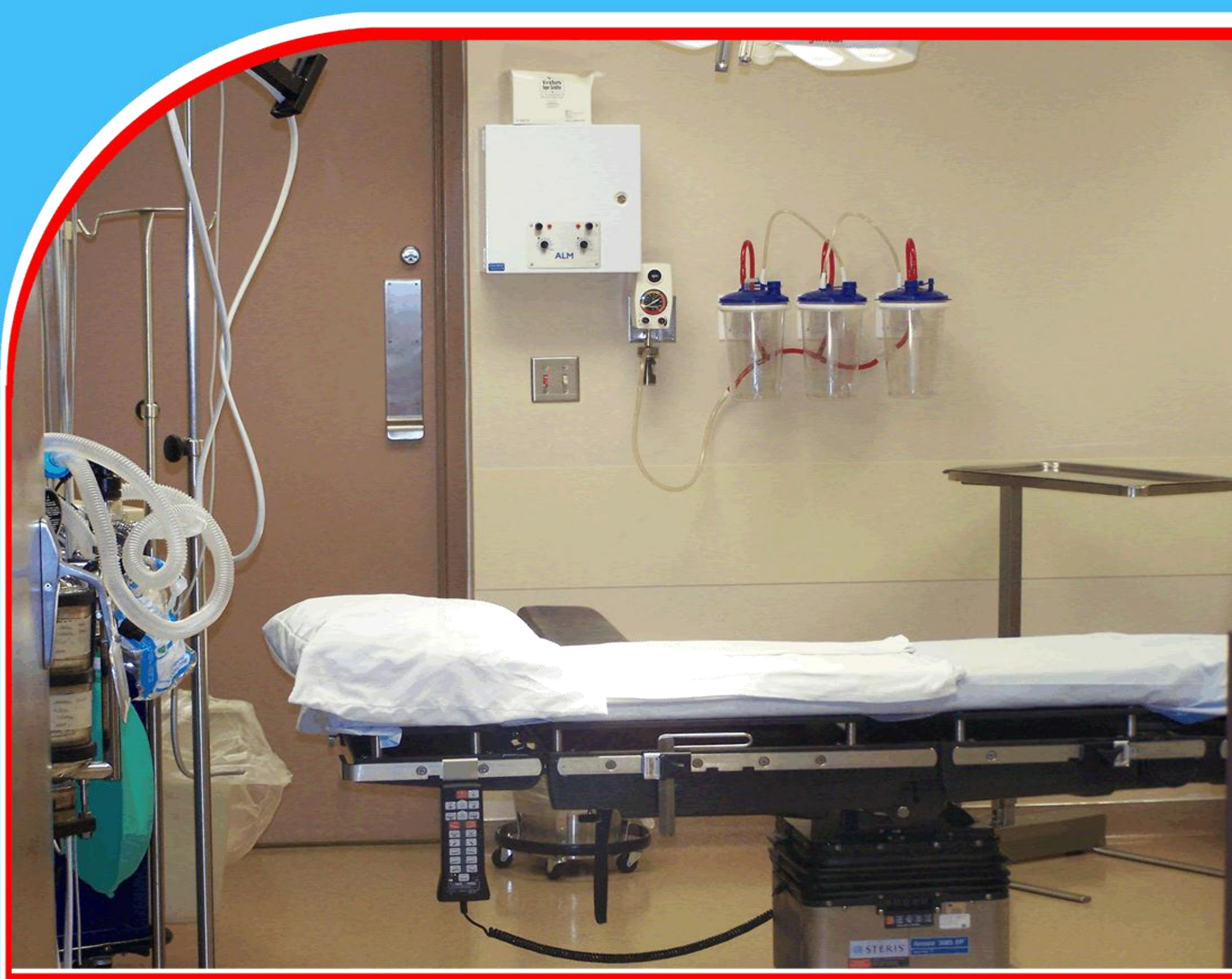

Outcome of acute kidney injuries following chronic kidney disease: A systematic review in Nawaz Sharif kidney hospital Swat

Rahmat Ali Khan, Syed Munib, Mohammad Shahzad, Mufti Baleegh, Liaqat Ali, Jehan Zeb, Mohammad Shahid, and Mohammad Asif

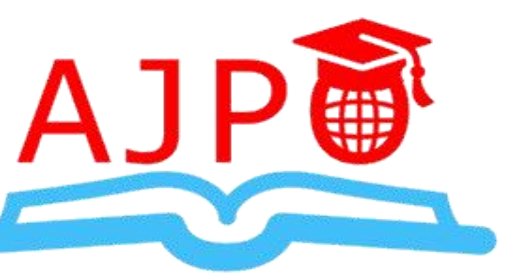




\title{
Outcome of acute kidney injuries following chronic kidney disease: A systematic review in Nawaz Sharif kidney hospital Swat
}

\author{
${ }^{1}$ Rahmat Ali Khan, ${ }^{2}$ Syed Munib, ${ }^{3}$ Mohammad Shahzad, ${ }^{4}$ Mufti Baleegh, ${ }^{5}$ Liaqat Ali, ${ }^{6}$ Jehan Zeb, \\ ${ }^{7}$ Mohammad Shahid, ${ }^{8}$ Mohammad Asif \\ ${ }^{1}$ Assistant Professor Nephrology, Head of Nephrology department, Nawaz Sharif Kidney Hospital, Swat, \\ Pakistan. \\ ${ }^{2}$ Associate Professor Nephrology Institute of Kidney Diseases (IKD), Peshawar, Pakistan. \\ ${ }^{3,5}$ Associate Professor Urologist Institute of Kidney Diseases (IKD), Peshawar, Pakistan. \\ ${ }^{4}$ Consultant Nephrologist Peshawar, Pakistan. \\ ${ }^{6}$ Consultant urologist institute of kidney diseases (IKD) Peshawar, Pakistan. \\ ${ }^{7}$ Consultant nephrologist dhq hospital Batkhala, Pakistan. \\ ${ }^{8}$ Consultant urologist dhq hospital Mardan, Pakistan.
}

Corresponding Authors: Syed Munib, Muhmmand shahzad, Liaqat ali

Emails: munibsyed@gmail.com, shahzadtauni@hotmail.com, liqat 99@yahoo.com

ABSTRACT

Background: Acute kidney injury may increase the risk for CKD and end-stage renal disease. In an attempt to summarize the literature and provide more compelling evidence, we conducted a systematic review comparing the risk for CKD (chronic kidney disease), AKI (Acute kidney injury), Acute gastroententeritis, postnatal AKI, Acute MI (myocardial infarction), AKI 2ndry to chemotherapy, AKI 2ndry to abstractive Nephropathy, AKI 2ndry to sepsis, AKI 2ndry to Drugs (NSAIDS and ARBS), AKI 2ndry to AGN (acute Glomerulonephritis), AKI 2ndry to Rhabdomyolysis, and lest AKI 2ndry to Malaria, death in patients with AKI,HD, CKD (chronic kidney disease). There have been several important developments in the literature recently regarding the association between acute kidney injury (AKI) and chronic kidney disease (CKD). First, when the National Kidney Foundation promulgated their highly influential Kidney Disease Outcomes Quality Initiative CKD guidelines in 2002, six chapters were devoted to the complications associated with decreased glomerular filtration rate (GFR) including hypertension, anemia, nutritional status, bone disease/disorders of calcium, and phosphorus metabolism, neuropathy

Objective: To study the outcome of Acute kidney injury following chronic kidney disease; systematic review. Methods: This was a prospective observational study from January 2018 to December 2020. Patients visiting department of Nephrology Nawaz Sharif Kidney center Swat, number of patients included study 351. All ages and both sexes were considered. Patients treated elsewhere or who has undergone in this study. Complete medical history, detailed examination like age, sex, diagnosis, and outcome, of AKI, examination under microscope and investigations, and necessary blood investigations were carried out.

Results: Three fifty one (351) patients were included in this study. The age distribution showed (75\%) patients between 40-95 years and 25(25\%) between 32-39 years. Mean age was 43 years with Standard Deviation of \pm 35.66 . Among 351 patients $162(45 \%)$ patients were male and $189(55 \%)$ patients were female. Duration of symptoms in 24(17\%) was <4 months and $112(75 \%)$ had $>4$ months, with mean of 4 months and SD \pm 2.315 . Total 351 participants AKI 45(12\%) postnatal AKI 33(9\%) Acute MI 18(6\%) AKI 2ndry chemotherapy 15(5.72\%) AKI 2ndry to abstractive Nephropathy 55 (14\%) AKI 2ndry to sepsis 63 (17\%) AKI 2ndry to Drugs (NSAIDS and ARBS) 54 (14\%) CKD (chronic kidney disease) 48 (15\%) AKI 2ndry to Rhabdomylysis $18(6 \%)$

Conclusions: The study concludes that the acute kidney injury following CKD systematic reviewing among patients presenting with AKI,CHD ,AKI sepsis, ,AKI Drugs(NSIAD) in local hospital settings This can be reduced with proper health education in general public regarding prevention of the disease and hence its complications.

Keywords: Outcome, Acute kidney injury, following CKD, systematic review, kidney hospital Swat. 
American Journal of Health, Medicine and Nursing Practice ISSN 2520-4017 (Online)

Vol.7, Issue 1, pp $11-17,2022$

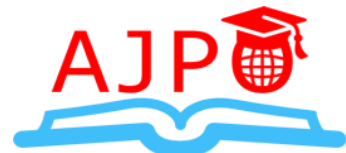

WWW.ajpojournals.org

\section{Introduction}

There have been several important developments in the literature recently regarding the association between acute kidney injury (AKI) and chronic kidney disease (CKD). First, when the National Kidney Foundation promulgated their highly influential Kidney Disease Outcomes Quality Initiative CKD guidelines in 2002, six chapters were devoted to the complications associated with decreased glomerular filtration rate (GFR) including hypertension, anemia, nutritional status, bone disease/disorders of calcium and phosphorus metabolism, neuropathy, and indices of functioning/well-being. ${ }^{1,}$ Notably, AKI was not included although it had long been known that patients with CKD were more prone to AKI ("acute on chronic renal failure"). Much of the CKD epidemiology literature around the time of and following the KDOQI CKD guideline publication focused on how reduced (estimated) GFR (and proteinuria) is related to risk of end-stage renal disease, cardiovascular events, and death. ${ }^{2-4}$ Since 2008 , however, a number of papers have sought to better quantify how the severity of CKD is a risk factor for development of AKI. ${ }^{5}$ These are discussed in more detail below (section "CKD as a risk factor for AKI").

Second, recent studies have highlighted the fact that the population incidence of AKI appears to be increasing rapidly. ${ }^{6-12}$ Recognizing the sizable and growing public health burden of AKI has focused more attention on its role in the natural history of CKD. ${ }^{13-14}$

Third, there has been a great deal of interest in and investigation into AKI as an instigator and promoter of CKD. Most of this manuscript will be devoted to reviewing the burgeoning literature on this topic. There is now a general consensus that AKI and CKD are, at times, two closely linked and interconnected syndromes. ${ }^{13.15 .16}$ Acute kidney injury may increase the risk for chronic kidney disease and end-stage renal disease. In an attempt to summarize the literature and provide more compelling evidence, we conducted a systematic review comparing therisk for AKI, CKD, AKI IM, SPSIS, AKI (NSIAD), and death in patients with and without AKI. From electronic databases, web search engines, and bibliographies, 13 cohort studies were selected, evaluatinglong-term renal outcomes and non-renal outcomes in patients with AKI. The pooled incidence of AKI and CKD were 25.8 per 100 person-years and 8.6 per 100 person-years,respectively. Patients with AKI had higher risks for developing CKD (pooled adjusted hazard ratio 8.8, 95\% CI3.1-25.5), ESRD (pooled adjusted HR 3.1, 95\% CI 1.9-5.0), and mortality (pooled adjusted HR 2.0, 95\% CI 1.3-3.1) comparedwith patients without AKI. The relationship between AKI and CKD or ESRD was graded on the basis of the severity of AKI, and the effect size was dampened by decreased baseline glomerular filtration rate. Data were limited, but AKI was also independently associated with the risk for cardiovascular disease and congestive heart failure, but not with hospitalization for stroke or all-cause hospitalizations. Meta-regression did not identify any study-level factors that were associated with the risk for AKI or CKD. Our review identifies AKI as an independent risk factor for AKI, CKD, AKI IM, SPSIS, AKI (NSIAD),death, and other important non-renal outcomes.

\section{METHODOLOGY}

This was a prospective observational study conducted at the department of Nephrology Nawaz Sharif kidney Hospital swat. The study period was from June 2018 to December 2020. For the purpose of the study, we included all patients of all ages from both genders the researchers above findings. Patient data was recorded, a pro-forma was made to collect all the necessary information. Detailed medical history was taken and AKI, CHD, AKI sepsis, AKI Drugs under microscope 
examination was carried out. AKI and computed CHD was done with required blood investigations. Patients treated elsewhere or who has undergone surgical procedure were excluded from the study. The study approval was obtained from the institutional review and ethical board before commencing the study. Patients were selected based on the above criteria from emergency, OPD and consultation calls. A detailed clinical history and examination was performed and final diagnosis was established. Data was collected regarding patient demographics such as age and gender, diagnosed cases of AKI, CHD, AKI Sepsis, Aki Drug (NSAID) all type were recorded separately and duration of symptoms in months was recorded. Data was stored in a pre-designed digital MS Excel chart and was analyzed in SPSS version 22. Data was represented as percentages and frequencies for categorical variables such as gender distribution and proportion. Continuous variables were presented as mean \pm standard deviation such as age and duration of symptoms. ChiSquare test was applied on post stratification of age and gender in which $\mathrm{P}$ Value $\leq 0.05$ was considered as significant value. Out of 351 patients, AKI 45(12\%) postnatal, AKI 33(9\%) Acute MI 18(6\%) AKI 2ndry chemotherapy 15(5.72\%) AKI 2ndry to abstractive Nephropathy 55 (14\%) AKI 2ndry to sepsis 63 (17\%) AKI 2ndry to Drugs (NSAIDS and ARBS) 54 (14\%) CKD (chronic kidney disease) 48 (15\%) AKI 2ndry to Rhabdomyolysis 18(6\%).

Table 1: Outcome baseline details of all the patients.

\begin{tabular}{lll}
\hline Variables & No. of Points & Percentage (\%) \\
\hline Age (Yrs) & 263 & \\
$40-95$ & 87 & $75 \%$ \\
31 to 40 & & $25 \%$ \\
Gender & 175 & \\
Male & 176 & $49.8 \%$ \\
Female & & $51.2 \%$ \\
Symptoms Duration & 24 & \\
$<4$ months & 351 & $12 \%$ \\
$>4$ months & & $88 \%$ \\
\hline
\end{tabular}


American Journal of Health, Medicine and Nursing Practice

ISSN 2520-4017 (Online)

Vol.7, Issue 1, pp $11-17,2022$

www.ajpojournals.org

Table 2: Comparison of patients' outcome of AKI, CKD diagnosis

\begin{tabular}{|c|c|c|c|c|c|}
\hline Patients diagnosis & No of pts & Male & Female & $\begin{array}{l}\text { Recovered/ } \\
\text { Expired }\end{array}$ & $\%$ age \\
\hline AKI (Acute kidney injury) & 45 & 25 & 20 & Recovered & $12 \%$ \\
\hline Postnatal AKI & 33 & 13 & 20 & Recovered & $9 \%$ \\
\hline Acute IM & 18 & 12 & 6 & Recovered & $6 \%$ \\
\hline Aki 2ndery chemotherapy & 15 & 6 & 9 & Recovered & $5.72 \%$ \\
\hline Aki 2ndery Abstractive Nephropathy & 45 & 25 & 20 & Recovered & $14 \%$ \\
\hline Aki 2ndery Sepsis & 53 & 30 & 23 & Recovered & 17 \\
\hline Aki 2ndery (NSAIDS+ARBS) & 46 & 20 & 28 & Recovered & 14 \\
\hline CKD chronic kidney disease & 48 & 24 & 24 & 22 Expired & 15 \\
\hline AKI 2ndery Rhabmylsis & 14 & 10 & 4 & Recovered & 6 \\
\hline AKI 2ndry to AGN & 26 & 6 & 20 & Recovered & $9 \%$ \\
\hline AKI 2ndry to Malaria & 8 & 4 & 4 & Recovered & $6 \%$ \\
\hline Total patients $(351)$ & 351 & 175 & 176 & 301 & $88 \%$ \\
\hline
\end{tabular}

Discussion

This is the first systematic review and meta-analysis summarizing the association of AKI with CKD. The data suggest that patients who survive AKI have a greater risk of CKD, and other adverse outcomes compared to patients without AKI after adjustment for several important confounding variables. The relationship between AKI and CKD was graded, with a greater risk associated with increasing severity of AKI. The risk for AKI was modified by baseline GFR and the risk for CKD was modified by pre-existing proteinuria. Recovery of AKI diminished the relationship between AKI, CKD. These findings that AKI increases the risk for CKD are not surprising. However, this meta-analysis provides an opportunity to systematically estimate the associated absolute and relative risk of these outcomes after AKI. One finding that is potentially counterintuitive is that patients with normal GFR prior to AKI had a higher relative risk for the development of CKD compared to those with AKI in the setting of decreased baseline GFR. For example, in the study by Wald et al., the absolute risk for CKD without AKI and decreased baseline GFR was $8.8 \%$ and increased to $17.2 \%$ for those with AKI and decreased baseline GFR (approximately 2-fold higher).

For those with normal baseline GFR, the absolute risks for CKD were $3.9 \%$ and $0.5 \%$ (more than 10-fold) for those with and without AKI, respectively. While the absolute risk for CKD was lower in those without decreased baseline GFR and AKI than those with AKI and decreased baseline GFR, the relative risk (hazard ratio) for CKD was greater in those with normal GFR because of the extremely low probability (denominator) of CKD in those without AKI or decreased baseline GFR. The reasons why AKI would increase the risk of CKD and other adverse outcomes remain unknown. Data from experimental animals suggests that AKI can induce renal fibrosis, and also 
that it can affect other organs in a deleterious fashion, such as the lungs, heart, and liver ${ }^{4,5}$. Thus, despite the fact that AKI is typically reversible in nature, by the standard of serum creatinine concentrations, there may be subclinical renal and extra-renal damage that persists and mediates these outcomes. However, despite all of the fact that all studies performed multivariable adjustments to derive the adjusted point estimates for the hazard ratios via a substantial number of demographic, physiologic and clinical variables, there still remains the possibility that the "independent relationship" between AKI and the outcomes is still biased by residual confounding due to unmeasured variables or imprecise measurement of known confounders. For example, several studies included in this systematic review ${ }^{11,14,16,17,20,22}$ used ICD-9 codes for ascertainment of important confounders such as CKD, diabetes, hypertension, and heart disease. Administrative codes have been shown to under-report co-morbidities. ${ }^{25}$

Furthermore, claims provide nothing in the context of the severity of the co-morbidity (e.g., dietcontrolled type 2 diabetes vs. severe type 2 diabetes requiring high doses of insulin with or without adequate glycemic control). Thus, in the absence of a randomized, controlled trial of an intervention that reduces AKI which in turn reduces the incidence of CKD or any other of the outcomes potentially related to AKI, then it is challenging to deduce causality with absolute confidence. The strengths of this systematic review are the following: adequate length of followup in the studies (most over 2 years), all studies reported parameter estimates of the hazard ratio adjusted for confounders, four studies examined outcomes associated with differing severities of AKI. The limitations include a high-degree of statistical heterogeneity among the primary analyses which may limit the validity of the point estimates and confidence intervals. However, we were able to demonstrate stable associations when more homogeneous studies were pooled. Part of the heterogeneity was related to different definitions of AKI (and of CKD). However, the outcomes of CKD and death are less prone to bias.

Since this review demonstrates an unequivocal association between AKI and CKD in a number of large, well-performed studies, we would encourage the medical community to focus efforts on determining whether this relationship is modifiable or simply a prognostic factor that serves as a barometer and predictor of ongoing and future risk. Only elegant studies in experimental animals and randomized trials in humans of interventions to prevent AKI prior to its occurrence or given after AKI to ameliorate the severity and duration of injury or interfere with subsequent organ injury will be able to address this question.

\section{Conclusions}

This study concludes that acute kidney injury following CKD the type of outcome CSOMis /Results $12 \%$ among patients presenting with chronic with AKI, CKD in local settings. This can be reduced with proper health education in general public regarding prevention of the disease and hence its complications. The study concludes that the acute kidney injury following CKD systematic reviewing among patients presenting in local hospital Study conducted the study show that 351 patients male $175(49.8 \%)$ and 176 female $(51.2 \%)$ total recovered 301 patients out of 351 and expire with CKD $22(6 \%)$ and recovery rate $(88 \%)$ in mention study This can be reduced with proper health education in general public regarding prevention of the disease and hence its complications. 
American Journal of Health, Medicine and Nursing Practice ISSN 2520-4017 (Online)

Vol.7, Issue 1, pp $11-17,2022$

www.ajpojournals.org

\section{References}

1. National Kidney Foundation. K/DOQI Clinical Practice Guidelines for Chronic Kidney Disease: Evaluation, Classification, and Stratification. Am J Kidney Dis. 2002;39(2 Suppl 1):S1S266. [PubMed] [Google Scholar]

2. Sarnak MJ, Levey AS, Schoolwerth AC, et al. Kidney disease as a risk factor for development of cardiovascular disease: a statement from the American Heart Association Councils on Kidney in Cardiovascular Disease, High Blood Pressure Research, Clinical Cardiology, and Epidemiology and Prevention. Circulation. 2003;108(17):2154-2169. [PubMed] [Google $\underline{\text { Scholar] }}$

3. Go A, Chertow G, Fan D, MuCulloch C, Hsu C. Chronic kidney disease and risks of death, cardiovascular events, and hospitalization. N Engl J Med. 2004;351:12961305. [PubMed] [Google Scholar]

4. Hemmelgarn BR, Manns BJ, Lloyd A, et al. Relation between kidney function, proteinuria, and adverse outcomes. JAMA. 2010;303(5):423-429. [PubMed] [Google Scholar]

5. Hsu CY, Ordonez JD, Chertow GM, Fan D, McCulloch CE, Go AS. The risk of acute renal failure in patients with chronic kidney disease. Kidney Int. 2008;74(1):101-107. [PMC free article] [PubMed] [Google Scholar]

6. Xue JL, Daniels F, Star RA, et al. Incidence and mortality of acute renal failure in Medicare beneficiaries, 1992 to 2001. J Am Soc Nephrol. 2006;17(4):1135-1142. [PubMed] [Google $\underline{\text { Scholar] }}$

7. Waikar SS, Curhan GC, Wald R, McCarthy EP, Chertow GM. Declining mortality in patients with acute renal failure, 1988 to 2002. J Am Soc Nephrol. 2006;17(4):1143-

1150. [PubMed] [Google Scholar]

8. Hsu CY, McCulloch CE, Fan D, Ordonez JD, Chertow GM, Go AS. Community-based incidence of acute renal failure. Kidney Int. 2007;72(2):208-212. [PMC free

article] [PubMed] [Google Scholar]

9. Hsu RK, McCulloch CE, Dudley RA, Lo LJ, Hsu CY. Temporal changes in incidence of dialysis-requiring AKI. J Am Soc Nephrol. 2013;24(1):37-42. [PMC free

article] [PubMed] [Google Scholar]

10. Wald R, McArthur E, Adhikari NK, et al. Changing incidence and outcomes following dialysis-requiring acute kidney injury among critically ill adults: a population-based cohort study. Am J Kidney Dis. 2015;65(6):870-877. [PubMed] [Google Scholar]

11. Siew ED, Davenport A. The growth of acute kidney injury: a rising tide or just closer attention to detail? Kidney Int. 2014 [PMC free article] [PubMed] [Google Scholar]

12. Hsu RK, McCulloch CE, Heung M, et al. Exploring potential reasons for the temporal trend in dialysis-requiring acute kidney injury in the United States. Clinical journal of the American Society of Nephrology: CJASN. 2016 in press. [PMC free article] [PubMed] [Google Scholar] 
American Journal of Health, Medicine and Nursing Practice ISSN 2520-4017 (Online)

Vol.7, Issue 1, pp $11-17,2022$

www.ajpojournals.org

13. Okusa MD, Chertow GM, Portilla D. The nexus of acute kidney injury, chronic kidney disease, and World Kidney Day 2009. Clinical journal of the American Society of Nephrology: CJASN. 2009;4(3):520-522. [PMC free article] [PubMed] [Google Scholar]

14. Hsu CY. Where is the epidemic in kidney disease? J Am Soc Nephrol. 2010;21(10):16071611. [PubMed] [Google Scholar]

15. Chawla LS, Kimmel PL. Acute kidney injury and chronic kidney disease: an integrated clinical syndrome. Kidney Int. 2012;82(5):516-524. [PubMed] [Google Scholar]

16. Chawla LS, Eggers PW, Star RA, Kimmel PL. Acute kidney injury and chronic kidney disease as interconnected syndromes. N Engl J Med. 2014;371(1):58-66. [PubMed] [Google Scholar]

17. Hsu RK, Hsu CY. Proteinuria and reduced glomerular filtration rate as risk factors for acute kidney injury. Curr Opin Nephrol Hypertens. 2011;20(3):211-217. [PMC free article] [PubMed] [Google Scholar] 